\title{
PERFIL E CONDIÇÃO BUCAL DO PACIENTE PORTADOR DE DOENÇAS INFECTOCONTAGIOSAS ATENDIDOS NO HOSPITAL OSWALDO CRUZ - CURITIBA/PR
}

\author{
Profile and oral condition of patients with infectious diseases attended in \\ Hospital Oswaldo Cruz - Curitiba/PR
}

\author{
Vinicius Villas Boas Petroni ${ }^{1}$ \\ Joslei Carlos Bohn² \\ Cassiano Lima Chaiben ${ }^{3}$ \\ Maria Helena Martins Tommasi ${ }^{4}$ \\ Ângela Fernandes ${ }^{5}$ \\ Francisca Berenice Dias Gil ${ }^{6}$ \\ Antonio Adilson Soares de Lima ${ }^{7}$
}

\begin{abstract}
RESUMO
A saúde bucal afeta a qualidade de vida e dignidade pessoal dos pacientes. Esse impacto parece ser mais grave em indivíduos medicamente comprometidos ou hospitalizados. O projeto intitulado "Atenção estomatológica em pacientes hospitalizados e portadores de doenças infectocontagiosas" desenvolveu atividades de atenção primária à saúde por meio de ações educativas e preventivas das doenças bucais nos indivíduos portadores de doenças infectocontagiosas que estavam em tratamento no Hospital Oswaldo Cruz (Curitiba/PR). Os indivíduos que participaram deste projeto foram submetidos a exame odontológico sob o auxílio de luz artificial nas enfermarias. Quando necessário, biópsias foram realizadas nos pacientes com lesões bucais sem diagnóstico clínico definitivo. 289 homens (65,38\%) e 153 mulheres $(34,62 \%)$ foram examinados. A média de idade dos indivíduos foi de 41 anos. As condições bucais mais frequentemente diagnosticadas nestes pacientes foram: doença periodontal, cárie, língua saburrosa e a candidose bucal. Este grupo de pacientes apresenta um risco elevado ao desenvolvimento de doenças bucais, especialmente as infecções oportunistas e as neoplasias. O diagnóstico precoce, o tratamento e a prevenção destas entidades promovem uma melhoria na saúde e na qualidade de vida destes indivíduos e o cirurgião-dentista desempenha um papel fundamental neste processo.
\end{abstract}

Palavras-chave: doenças transmissíveis; saúde bucal; hospitalização; unidade hospitalar de odontologia; síndrome da imunodeficiência adquirida.

\footnotetext{
${ }_{1}^{1}$ Aluno do Curso de graduação em Odontologia da UFPR - E-mail vinicius_petroni@hotmail.com

2 Aluno do Curso de graduação em Odontologia da UFPR - E-mail josleibohn@hotmail.com

${ }^{3}$ Aluno do Programa de Pós-graduação em Odontologia da UFPR - E-mail cassianochaiben@gmail.com

${ }^{4}$ Departamento de Estomatologia do Curso de Odontologia da UFPR - E-mail mhtommasi@yahoo.com.br

${ }^{5}$ Departamento de Estomatologia do Curso de Odontologia da UFPR - E-mail angelfnandes@hotmail.com

${ }^{6}$ Hospital Oswaldo Cruz - SESA - E-mail gil.francisca@hotmail.com

${ }^{7}$ Departamento de Estomatologia do Curso de Odontologia da UFPR - E-mail antollima@hotmail.com
}

PETRONI, Vinicius V.B. et all. Perfil e condição bucal do paciente portador de doenças infectocontagiosas atendidos no Hospital Oswaldo Cruz - Curitiba/PR. Extensão em Foco, Curitiba: Ed. da UFPR, nr.9, jan/jun 2014, p.94-105. ISSN 2358-7180. 


\begin{abstract}
Oral health affects quality of life and personal dignity of patients. This impact appears to be more severe in hospitalized or medically compromised individuals. The project titled "Stomatological attention to hospitalized patients and patients with infectious diseases" developed activities of primary health care through educational and prevention of oral diseases with individuals who have got infectious diseases and that were undergoing treatment at the Hospital Oswaldo Cruz (Curitiba/PR). Individuals who participated in this project underwent dental examination under the aid of artificial light in wards. When necessary, biopsies were performed in patients with oral lesions without a definite clinical diagnosis. 289 men (65.38\%) and 153 women (34.62\%) were examined. The subjects' mean age was 41 years. The most frequently diagnosed oral conditions in these patients were: periodontal diseases, cavities, coated tongue and oral candidosis. This group of patients have a high risk for the development of oral diseases, especially opportunistic infections and neoplasms. Early diagnosis, treatment and prevention of these entities promote improvement in the health and quality of life of those individuals and the dental surgeon plays a key role in this process.

Keywords: communicable diseases; oral health; hospitalization; dental service hospital; acquired immune deficiency syndrome.
\end{abstract}

\title{
Introdução
}

A atuação do cirurgião-dentista em ambiente hospitalar envolve a realização de procedimentos que vão além das proporções imaginadas e atribuídas pela população, uma vez que os procedimentos realizados não dizem respeito somente às intervenções cirúrgicas (GODOI et al., 2009). A Odontologia hospitalar é descrita como uma prática que visa os cuidados das alterações bucais que exigem procedimentos de equipes multidisciplinares de alta complexidade ao paciente (CAMARGO, 2005). Entretanto, na atualidade, este conteúdo tem sido negligenciado no currículo acadêmico da maioria das universidades brasileiras.

Neste contexto, os cirurgiões-dentistas precisam estar integrados numa equipe multidisciplinar e estes profissionais devem ter em mente que a sua abordagem envolverá o paciente como um todo e não somente nos aspectos relacionados aos cuidados com a boca (GODOI et al., 2009). Desta forma, o cirurgião-dentista que presta atendimento a pacientes hospitalizados precisa estar apto a executar as seguintes funções: a) Realização de procedimentos emergenciais (dores de dente, sangramentos, feridas na boca, controle da halitose, etc.); b) Emissão de resposta a pareceres sobre alterações

PETRONI, Vinicius V.B. et all. Perfil e condição bucal do paciente portador de doenças infectocontagiosas atendidos no Hospital Oswaldo Cruz - Curitiba/PR. Extensão em Foco, Curitiba: Ed. da UFPR, nr.9, jan/jun 2014, p.94-105. ISSN 2358-7180. 
estomatológicas; c) Promoção de atendimento complexo em hospital (cirúrgico ou clínico) aos pacientes com necessidades especiais ou nos casos onde há a necessidade do uso de sedação e/ou anestesia geral; d) Capacitação e acompanhamento de equipes auxiliares para a manutenção da saúde bucal de pacientes internados; e) Incentivo à higienização bucal com métodos mecânicos (escovação dentária, lingual e uso adequado do fio dental) e ainda o uso de técnicas especiais de profilaxia (limpeza) dentária e periodontal; f) 0 uso correto de enxaguatórios para remoção da placa bacteriana bucal e umedecimento das mucosas; g) constante inspeção da boca e estruturas associadas; e h) criação de protocolos para notificação do cirurgião-dentista em caso de alterações nas estruturas bucais e adjacentes.

A higiene bucal de rotina para pacientes hospitalizados é imperativa e os cuidados com a saúde bucal é uma parte importante do tratamento. Entretanto, esta ação geralmente tem ficado sob a responsabilidade de enfermeiros sem conhecimento suficiente em higiene bucal ou protocolos abrangentes para seguir (EDWARD et al., 2012).

Atualmente, sabe-se que a efetividade dos cuidados em saúde bucal realizados em pacientes com desordens neurocirúrgicas por higienistas dentais, em colaboração com as enfermeiras, desempenha um papel importante na promoção da saúde não apenas bucal, mas também na saúde geral do paciente (MORI et al., 2012).

Os hospitais de grande porte tendem a ser mais interessados em oferecer cuidados de saúde bucal do que os hospitais menores. A realização de cuidados com a saúde bucal já é comum em hospitais de alguns países e há uma grande demanda pelo treinamento de técnicas de higiene bucal e na obtenção de informações por meio dos cirurgiões-dentistas. É importante promover a cooperação entre o pessoal da enfermagem e os cirurgiõesdentistas dentro de hospitais em relação à higiene bucal (KURAMOTO et al., 2011). Ao ser internado, o paciente poderá ter a sua saúde bucal seriamente comprometida em virtude da própria doença de base ou dos efeitos colaterais de diversos medicamentos que o mesmo está fazendo uso como parte do tratamento. Desta forma, a hipossalivação, as infecções oportunistas na boca,

PETRONI, Vinicius V.B. et all. Perfil e condição bucal do paciente portador de doenças infectocontagiosas atendidos no Hospital Oswaldo Cruz - Curitiba/PR. Extensão em Foco, Curitiba: Ed. da UFPR, nr.9, jan/jun 2014, p.94-105. ISSN 2358-7180. 
as lesões de cárie, a doença periodontal, a halitose e a língua saburrosa acabam sendo achados comuns em indivíduos hospitalizados. Além disso, o paladar fica prejudicado principalmente em pacientes idosos (SOLEMDAL et al., 2012).

Atualmente, sabe-se que os pacientes com doenças infectocontagiosas, especialmente a AIDS, podem apresentar uma série de alterações bucais decorrentes do grau de comprometimento do sistema imunológico. Dávila e Gil (2011) descreveram que as manifestações bucais mais frequentes nestes pacientes são representadas pela candidose bucal, queilite angular, eritema gengival linear e os aumentos de volume nas glândulas parótidas. Desta forma, a criação de estratégias focadas no manejo preventivo e terapêutico das manifestações bucais é fundamental para melhorar a saúde e a qualidade de vida destes pacientes.

Com vistas à promoção da saúde bucal aos indivíduos hospitalizados decorrentes de complicações do quadro clínico de doenças infectocontagiosas, tornou-se necessário conhecer o perfil deste tipo de pacientes. Sendo assim, o projeto intitulado "Atenção odontológica em indivíduos portadores de doenças infectocontagiosas" ( $N^{\circ}$ de registro na PROEC: 54409) foi aprovado em 2009 e tinha como objetivo principal o desenvolvimento de atividades de atenção primária à saúde bucal com ações voltadas à prevenção e ao tratamento de doenças bucais em indivíduos com doenças infectocontagiosas que se encontravam internados no Hospital Oswaldo Cruz, Curitiba/PR. Além disso, o projeto permitiu a participação de alunos da graduação e da pós-graduação em suas ações, de forma a incluir em sua formação uma postura de inserção social e cidadã, integrada às atividades de ensino, pesquisa e extensão. O objetivo deste artigo é relatar o perfil e as condições bucais dos pacientes que foram atendidos no projeto de extensão "Atenção odontológica em indivíduos portadores de doenças infectocontagiosas".

\section{Metodologia}

O projeto "Atenção estomatológica em pacientes hospitalizados e portadores de doenças infecto-contagiosas" desenvolveu atividades de atenção

PETRONI, Vinicius V.B. et all. Perfil e condição bucal do paciente portador de doenças infectocontagiosas atendidos no Hospital Oswaldo Cruz - Curitiba/PR. Extensão em Foco, Curitiba: Ed. da UFPR, nr.9, jan/jun 2014, p.94-105. ISSN 2358-7180. 
primária à saúde com ações educativas voltadas à prevenção de doenças bucais em indivíduos portadores de doenças infectocontagiosas (Tuberculose, AIDS, Toxoplasmose, Sífilis, entre outras) que estavam em leito hospitalar ou em tratamento ambulatorial. Este tipo de paciente apresenta um risco elevado ao desenvolvimento de doenças bucais, especialmente as infecções oportunistas, como a cárie, a doença periodontal e as neoplasias bucais.

A metodologia desenvolvida implicou no desenvolvimento das seguintes ações: a) Realização de exame clínico odontológico; b) Orientação da higiene bucal; c) Execução de biópsia para a confirmação do diagnóstico de lesões bucais; d) Tratamento das lesões das estruturas bucais e peribucais; e) Encaminhamento dos pacientes que necessitavam de tratamento específico ou especializado; e f) Levantamento epidemiológico das doenças bucais e peribucais.

Este exame odontológico do paciente foi feito por meio da palpação e inspeção da boca usando luz artificial, espátula de madeira, compressas de gaze e equipamentos de proteção individual. Os dados pessoais e da história da doença atual foram coletados e anotados numa planilha.

Quando necessário, os pacientes foram submetidos à biópsia, tratados com a prescrição de medicamentos e/ou encaminhados para tratamento especializado na clínica do Curso de Odontologia da UFPR.

Ao final de cada atendimento, a equipe de professores e alunos se reunia juntamente com a cirurgiã-dentista e com os médicos para discutir os detalhes dos casos. Além disso, a direção do hospital permitiu que os membros deste projeto assistissem mensalmente às palestras que foram apresentadas pelo corpo clínico sobre doenças infectocontagiosas.

Por último, os dados dos pacientes foram tabulados e submetidos à estatística descritiva.

\section{Resultados}

A maioria dos indivíduos que foi atendida pelo projeto de extensão era do sexo masculino - 289 homens (65,38\%) e 153 mulheres (34,62\%). A média de idade dos indivíduos foi de 41 anos. A média de idade para os homens foi

PETRONI, Vinicius V.B. et all. Perfil e condição bucal do paciente portador de doenças infectocontagiosas atendidos no Hospital Oswaldo Cruz - Curitiba/PR. Extensão em Foco, Curitiba: Ed. da UFPR, nr.9, jan/jun 2014, p.94-105. ISSN 2358-7180. 
de 41 anos e de 40 anos para as mulheres. Ainda em relação à faixa etária, verificou-se que a mesma foi distribuída entre os 19 e 84 anos. A tabela 1 apresenta a distribuição dos pacientes atendidos segundo a faixa etária.

Tabela 1 - Distribuição dos pacientes atendidos no Hospital Oswaldo Cruz segundo a idade. Curitiba/PR. 2012.

\begin{tabular}{ccc} 
Faixa etária & Pacientes & Porcentagem \\
\hline $19-29$ anos & 73 & $16,5 \%$ \\
\hline $30-59$ anos & 345 & $78,0 \%$ \\
\hline Acima de 60 anos & 24 & $5,5 \%$ \\
\hline Total & 442 & $100 \%$ \\
\hline
\end{tabular}

Fonte: Dados do projeto

A maioria dos pacientes que foram atendidos no projeto estavam internados no hospital devido a complicações da infecção HIV/AIDS. As tabelas 2 e 3 apresentam a distribuição das lesões bucais observadas nos pacientes com HIV/AIDS segundo a classificação EC-Clearinghouse desenvolvida para esta doença.

Tabela 2 - Distribuição das lesões bucais fortemente associadas à infecção pelo HIV segundo a Classificação EC-Clearinghouse. Curitiba/PR. 2012.

\begin{tabular}{c|c|c}
\hline Lesão & N & \% \\
\hline Candidoses & 184 & $28,09 \%$ \\
\hline Doença Periodontal & 157 & $23,97 \%$ \\
\hline Leucoplasia Pilosa Oral & 033 & $5,04 \%$ \\
\hline Sarcoma de Kaposi & 003 & $0,46 \%$ \\
\hline Linfoma Não Hodgkin & 002 & $0,31 \%$
\end{tabular}

Fonte: Dados do projeto

Tabela 3 - Distribuição das lesões bucais menos comumente associadas à infecção pelo HIV segundo a Classificação EC-Clearinghouse. Curitiba/PR. 2012.

\begin{tabular}{|c|c|c|}
\hline Lesão & $\mathbf{N}$ & $\%$ \\
\hline Hiperpigmentação Melânica & 034 & $5,19 \%$ \\
\hline
\end{tabular}

PETRONI, Vinicius V.B. et all. Perfil e condição bucal do paciente portador de doenças infectocontagiosas atendidos no Hospital Oswaldo Cruz - Curitiba/PR. Extensão em Foco, Curitiba: Ed. da UFPR, nr.9, jan/jun 2014, p.94-105. ISSN 2358-7180. 


\begin{tabular}{c|c|c}
\hline Estomatite Ulcerativa Necrosante & 001 & $0,15 \%$ \\
\hline Doenças das Glândulas Salivares & 001 & $0,15 \%$ \\
\hline Púrpura Trombocitopênica & 000 & - \\
\hline Ulcerações Inespecíficas & 039 & $5,95 \%$ \\
\hline Infecções Virais & 034 & $5,19 \%$ \\
\hline
\end{tabular}

Fonte: Dados do projeto

O exame clínico dos pacientes revelou que os principais problemas bucais que estes indivíduos apresentavam era a presença de lesões de cárie e de cálculo. Este último está associado à etiopatogenia da doença periodontal. A tabela 4 apresenta os dados relacionados às condições bucais dos pacientes hospitalizados.

Tabela 4 - Distribuição das condições dentárias dos pacientes internados no Hospital Oswaldo Cruz. Curitiba/PR. 2012.

\begin{tabular}{l|c|c}
\hline Condições Dentárias & $\mathbf{n}$ & $\%$ \\
\hline Cáries & 190 & $29,01 \%$ \\
\hline Raíz(es) Residual(is) & 131 & $20,00 \%$ \\
\hline Cálculos/Indutos & 165 & $25,19 \%$ \\
\hline Dente Fraturado & 025 & $3,82 \%$ \\
\hline Desgastes Incisais/em cúspides & 016 & $2,44 \%$ \\
\hline Dente com Curativo Endodôntico & 014 & $2,14 \%$ \\
\hline Restaurações em Amálgama & 086 & $13,13 \%$ \\
\hline Restaurações em Resina Composta & 017 & $2,60 \%$ \\
\hline Uso de próteses & & \\
\hline Ausências Dentárias & 157 & $23,97 \%$ \\
\hline Prótese Total & 032 & $4,89 \%$ \\
\hline Prótese Parcial Removível & 021 & $3,21 \%$ \\
\hline Prótese Fixa & 002 & $0,31 \%$ \\
\hline Oclusão & \multicolumn{2}{|l}{} \\
\hline Apinhamento Dentário & 014 & $2,14 \%$ \\
\hline Mordida Cruzada & 001 & $0,15 \%$ \\
\hline Aparelho Ortodôntico fixo/móvel & $0,92 \%$ \\
\hline
\end{tabular}

PETRONI, Vinicius V.B. et all. Perfil e condição bucal do paciente portador de doenças infectocontagiosas atendidos no Hospital Oswaldo Cruz - Curitiba/PR. Extensão em Foco, Curitiba: Ed. da UFPR, nr.9, jan/jun 2014, p.94-105. ISSN 2358-7180. 
Fonte: Dados do projeto

As tabelas 5 e 6 exibem a distribuição do número de lesões e alterações dentárias e nos tecidos moles, respectivamente, que foram diagnosticadas nos pacientes após o exame odontológico. Os resultados demonstraram que os problemas dentários foram mais frequentes do que os problemas envolvendo a saúde dos tecidos moles.

Tabela 5 - Distribuição do número de alterações dentárias identificadas nos pacientes internados no Hospital Oswaldo Cruz. Curitiba/PR. 2012.

\begin{tabular}{|l|c|}
\hline Número de Alterações nos dentes & $\begin{array}{c}\text { Pacientes do Hospital Oswaldo Cruz } \\
\mathbf{n}(\%)\end{array}$ \\
\hline Sem lesões ou alterações & $119(55,09 \%)$ \\
\hline 1 alteração & $64(29,62 \%)$ \\
\hline 2 alterações & $25(11,57 \%)$ \\
\hline 3 alterações & $7(3,24 \%)$ \\
\hline$\geq 4$ alterações & $1(0,46 \%)$ \\
\hline Total & $\mathbf{2 1 6 ( 1 0 0 \% )}$ \\
\hline
\end{tabular}

Fonte: Dados do projeto

Tabela 6 - Distribuição do número de alterações na mucosa bucal diagnosticadas nos pacientes internados no Hospital Oswaldo Cruz. Curitiba/PR. 2012.

\begin{tabular}{|l|c|}
$\begin{array}{l}\text { Número de Alterações e Lesões da } \\
\text { Mucosa. }\end{array}$ & $\begin{array}{l}\text { Pacientes do Hospital Oswaldo Cruz } \\
\mathbf{n}(\%)\end{array}$ \\
\hline Sem Lesões ou Alterações & $20(9,25 \%)$ \\
\hline 1 Alteração & $88(40,74 \%)$ \\
\hline 2 Alterações & $76(35,18 \%)$ \\
\hline 3 Alterações & $22(10,18 \%)$ \\
\hline$\geq 4$ Alterações & $10(4,62 \%)$ \\
\hline Total & $\mathbf{2 1 6 ( 1 0 0 \% )}$ \\
\hline
\end{tabular}

Fonte: Dados do projeto

\section{Discussão}

A extensão universitária ou acadêmica é uma ação de uma Universidade junto à comunidade, disponibilizando ao público externo o conhecimento adquirido com o ensino e a pesquisa por ela desenvolvidos. Essa ação produz um novo conhecimento a ser trabalhado e articulado. Este artigo apresenta os

PETRONI, Vinicius V.B. et all. Perfil e condição bucal do paciente portador de doenças infectocontagiosas atendidos no Hospital Oswaldo Cruz - Curitiba/PR. Extensão em Foco, Curitiba: Ed. da UFPR, nr.9, jan/jun 2014, p.94-105. ISSN 2358-7180. 
dados relativos ao perfil e das condições bucais dos pacientes que foram atendidos no projeto de extensão "Atenção odontológica em indivíduos portadores de doenças infectocontagiosas" nos anos de 2009 a 2011.

Os resultados do presente projeto revelaram que a maioria dos indivíduos estavam internados devido às complicações da infecção HIV/AIDS. O perfil dos pacientes era do sexo masculino e com a idade entre 39 e 50 anos. Este também tem sido o perfil que se reproduz na maioria dos estudos realizados em outras partes do mundo.

Vários estudos demonstraram que 40-50\% dos indivíduos com HIV/AIDS têm infecções fúngicas, bacterianas ou virais na boca, que são susceptíveis de ocorrer no início do curso da doença (KHAN et al., 2012). No presente estudo, as infecções fúngicas seguidas das doenças que comprometem a saúde do periodonto foram as lesões bucais mais observadas na boca dos pacientes do Hospital Oswaldo Cruz. Atualmente, a infecção pelo HIV/AIDS é considerada como um problema devastador de saúde mundial, colocando desafios graves em países de baixa e média renda. O contínuo aumento do número de pessoas vivendo com HIV/AIDS representa um problema de saúde e econômico graves que o mundo está enfrentando (KHAN et al., 2012).

A Organização Mundial da Saúde fez considerações sobre a situação global atual das doenças que afetam a boca no documento intitulado Oral Health for the 21st Century, e também num relatório técnico, Recent Advances in Oral Health (1992). Esses relatórios examinaram as tendências e os avanços nas pesquisas em saúde bucal, a distribuição de cuidados com a saúde bucal, além da educação dos profissionais envolvidos com saúde bucal para o estabelecimento de mudanças de atitudes e necessidades dos membros da comunidade. A conclusão foi a de que os serviços de saúde bucal e a educação do pessoal devem ser radicalmente transformados (MURRAY, 2005).

As doenças da boca representam um problema de saúde importante. Elas são bastante frequentes e seu impacto, tanto na sociedade, quanto no indivíduo, é significativo. A dor, a incapacidade e a incompetência são características comuns nas doenças bucais. Além disso, os custos do tratamento são o principal fardo dos sistemas de saúde.

PETRONI, Vinicius V.B. et all. Perfil e condição bucal do paciente portador de doenças infectocontagiosas atendidos no Hospital Oswaldo Cruz - Curitiba/PR. Extensão em Foco, Curitiba: Ed. da UFPR, nr.9, jan/jun 2014, p.94-105. ISSN 2358-7180. 
A causa da maioria das doenças bucais é conhecida e as condições desencadeantes são amplamente evitáveis. De acordo com estes critérios, as doenças que afetam a boca e os dentes são um problema de saúde pública. Adicionalmente, as desigualdades na área da saúde bucal são um problema; os grupos populacionais excluídos e desfavorecidos socialmente sofrem com as maiores taxas da doença. Sendo assim, a educação em saúde bucal visa promover melhor conhecimento e a consciência em saúde bucal, por meio de ações educacionais e de divulgação de informações. A população, por meio da aquisição de conhecimento, provavelmente promoverá mudanças no seu comportamento. Todo projeto de educação em saúde visa atingir a coletividade e, consequentemente, uma mudança de atitudes.

A maioria dos pacientes que se encontravam hospitalizados não apresentava higiene oral satisfatória. A doença cárie e as doenças que afetam o periodonto estão associadas com os comportamentos de saúde. Além disso, um tempo maior no hospital está associado a níveis aumentados de inflamação gengival e no acúmulo de placa dental (CARRILHO NETO et al., 2011).

$O$ presente projeto de extensão desenvolveu um estudo realizado em indivíduos hospitalizados que revelou que $45.9 \%$ dos pacientes, principalmente aqueles de idade avançada, apresentavam uma ou mais lesões bucais. A xerostomia $(58.6 \%)$, a língua saburrosa $(54.1 \%)$ e a halitose $(46.8 \%)$ foram os achados bucais mais frequentemente observados (LOCKER et al., 2002).

As doenças da boca, em especial, a cárie, a doença periodontal e as infecções bucais continuam tendo alta prevalência nos dias de hoje. Medidas preventivas e educacionais são importantes na prevenção e para o diagnóstico precoce destas doenças. Este projeto possibilitará uma atuação junto à comunidade, pois levará orientações sobre as alternativas para melhorar a saúde bucal. Possibilitará, aos alunos envolvidos, a capacitação em trabalhos de campo e reflexões sobre os saberes científicos e empíricos introduzidos durante a interação com a comunidade.

O envolvimento universitário nesta realidade é extremamente importante, pois proporciona um ganho acadêmico aos participantes e promove mudanças na vida da comunidade envolvida. Os alunos da graduação que

PETRONI, Vinicius V.B. et all. Perfil e condição bucal do paciente portador de doenças infectocontagiosas atendidos no Hospital Oswaldo Cruz - Curitiba/PR. Extensão em Foco, Curitiba: Ed. da UFPR, nr.9, jan/jun 2014, p.94-105. ISSN 2358-7180. 
participaram do projeto haviam cursado recentemente a disciplina de semiologia aplicada e nela eles aprenderam a identificar os sinais e os sintomas das doenças bucais e peribucais visando à obtenção do diagnóstico. O envolvimento dos discentes nas ações extensionistas proporcionou-lhes a oportunidade de conhecer uma realidade que eles não encontram nas atividades acadêmicas do curso de Odontologia.

Neste projeto, os alunos, inicialmente, prestaram atendimento nas enfermarias sob a forma de realização do exame clínico bucal e biópsias com vistas ao diagnóstico. Este exame era feito por meio da palpação e inspeção usando luz artificial e na própria enfermaria. Esta experiência permitiu que o aluno da graduação pudesse realizar o exame de um paciente sistemicamente comprometido. Esta experiência não é um fato comum durante o atendimento das clínicas do curso regular de odontologia.

Além disso, os alunos e o orientador discutiam semanalmente os casos com os demais membros do corpo clínico do hospital. Muitas vezes, a discussão era realizada em conjunto com uma cirurgiã-dentista, com os médicos, os enfermeiros e as assistentes sociais do hospital. No momento em que o diagnóstico bucal era estabelecido, o paciente era tratado ou encaminhado para tratamento especializado. Ações preventivas em relação à saúde bucal eram ofertadas aos pacientes e, em algumas situações, aos seus acompanhantes (técnica de escovação dos dentes e da língua, assim como a higienização de próteses e dentaduras). Ao que se sabe, a hospitalização está associada a uma deterioração na saúde bucal, particularmente em pacientes intubados (SJÖGREN, 2011).

\section{REFERÊNCIAS}

CAMARGO, E. C. Odontologia hospitalar é mais do que cirurgia bucomaxilofacial. Jornal do Site, ano VI (98), 2005. Disponível em: $<$ http://www.jornaldosite.com.br/arquivo/anteriores/elainecamargo/artelainecam argo98.htm>. Acesso em: 20/10/2012.

CARRILHO NETO, A.; DE PAULA RAMOS, S.; SANT'ANA, A. C.; PASSANEZI, E. Oral health status among hospitalized patients. Int J Dent Hyg., v. 9, n. 1, p. 21-9, Feb. 2011.

PETRONI, Vinicius V.B. et all. Perfil e condição bucal do paciente portador de doenças infectocontagiosas atendidos no Hospital Oswaldo Cruz - Curitiba/PR. Extensão em Foco, Curitiba: Ed. da UFPR, nr.9, jan/jun 2014, p.94-105. ISSN 2358-7180. 
DÁVILA, M. E.; GIL, M. Oral manifestations and dental caries in children exposed to human immunodeficiency virus. Rev. Salud Pública (Bogotá), v. 13, n. 5, p. 833-843, Oct. 2011.

EDWARD, K. L.; FELSTEAD, B.; MAHONEY, A. M. Hospitalized mental health patients and oral health. J. Psychiatr. Ment. Health Nurs., v. 19, n. 5, p. 419425, Jun. 2012.

GODOI, A. P. T.; FRANCESCO, A. R.; DUARTE, A.; KEMP, A. P. T.; SILVALOVATO, C. H. Hospital odontology in Brazil. A general vision. Rev. Odontol. UNESP, v. 38, n. 2, p. 105-109, 2009.

KURAMOTO, C.; WATANABE, Y.; TONOGI, M.; HIRATA, S.; SUGIHARA, N.; ISHII, T.; YAMANE, G. Y. Factor analysis on oral health care for acute hospitalized patients in Japan. Geriatr Gerontol Int., v. 11, n. 4, p. 460-466, Oct. 2011.

KHAN, S. A.; MOORTHY, J.; OMAR, H.; HASAN, S. S. People living with HIV IAIDS (PLWHA) and HIV/AIDS associated oral lesions. A study in Malaysia. BMC Public Health, v. 12, n. 1, p. 850, Oct. 2012.

LOCKER, D.; MATEAR, D.; STEPHENS, M.; JOKOVIC, A. Oral health-related quality of life of a population of medically compromised elderly people. Community Dent Health, v. 19, n. 2, p. 90-97, 2002.

MORI, C.; HAKUTA, C.; ENDO, K.; NARIAI, T.; UENO, M.; SHINADA, K.; KAWAGUCHI, Y. The effects of professional oral health care on patients in the sub-acute stage of emergent neurosurgical disorders. Spec. Care Dentist, v. 32, n. 6, p. 259-264, Nov. 2012.

MURRAY, J. A saúde bucal no século XXI. In: MURRAY, J. J.; NUNN, J. H.; STEELE, J. G. Doenças orais. Medidas preventivas. 4. ed. Rio de Janeiro: Guanabara Koogan, 2005. p. 3-5.

SJÖGREN, P. Hospitalization associated with deterioration in oral health. Evid Based Dent., v. 12, n. 2, p. 48, 2011.

SOLEMDAL, K.; SANDVIK, L.; WILLUMSEN, T.; MOWE, M.; HUMMEL, T. The impact of oral health on taste ability in acutely hospitalized elderly. PLOS One, v. 7, n. 5, p. e36557, 2012.

PETRONI, Vinicius V.B. et all. Perfil e condição bucal do paciente portador de doenças infectocontagiosas atendidos no Hospital Oswaldo Cruz - Curitiba/PR. Extensão em Foco, Curitiba: Ed. da UFPR, nr.9, jan/jun 2014, p.94-105. ISSN 2358-7180. 